\title{
Social Isolation in the Rat Produces Developmentally Specific Deficits in Prepulse Inhibition of the Acoustic Startle Response Without Disrupting Latent Inhibition
}

Lawrence S. Wilkinson, Ph.D., Simon S. Killcross, Ph.D., Trevor Humby, B.Sc., Frank S. Hall, B.A., Mark A. Geyer, Ph.D., and Trevor W. Robbins, Ph.D.

A series of experiments examined the effects of 8 weeks of social isolation on spontaneous locomotor activity, prepulse inhibition (PPl) of the acoustic startle response, latent inhibition (LI) in a conditioned suppression paradigm, and basal and d-amphetamine stimulated dopamine $(D A)$ release in the ventral striatum, as measured by in vivo microdialysis. Both isolation-reared animals (those isolated from the weaning age) and isolation-housed animals (those isolated as adults) were hyperactive when placed in a novel environment. Social isolation also led to deficits in PPI of the acoustic startle response that were specific to isolation-reared animals. Isolation rearing was without effect on the expression of LI but did lead to an enhanced response to systemic $\mathrm{d}$-amphetamine in terms of striatal DA release. The data are discussed with respect to the involvement of ventral striatal DA mechanisms in the expression of PPI and LI, differences in the impact of social isolation in young and adult animals, and the utility of social isolation model as a nonlesion, nonpharmacologic means of perturbing ventral striatal DA function.

[Neuropsychopharmacology 10:61-72, 1994]
KEY WORDS: Social isolation; Locomotor activity;

Prepulse inhibition; Latent inhibition; Dopamine; Ventral striatum

Variations in early social experience have been shown to have profound effects on the adult behavior, brain morphology, and neurochemistry of a variety of species (Sachser 1986; Lewis et al. 1990; Martin et al. 1991; Jones et al. 1992). For example, rearing rats in social isolation from the weaning age produces behavioral abnormalities that include increases in spontaneous and

From the Department of Experimental Psychology (LSW, ASK, TH, FSH, TWR), University of Cambridge, Cambridge, United Kingdom and Department of Psychiatry (MAG), University of California at San Diego, La Jolla, California.

Address correspondence to: Lawrence S. Wilkinson, Ph.D., Department of Experimental Psychology, University of Cambridge, Downing Street, Cambridge, United Kingdom CB2 3EB.

Received July 7, 1993; revised October 19, 1993; accepted October 23, 1993. conditioned locomotor activity (Gentsch et al. 1981; Sahakian et al., 1982; Jones et al., 1990), altered responses to environmental novelty (Sahakian et al. 1977; Gentsch et al. 1982a, 1982b, 1983), impairments in discrimination learning (Jones et al. 1991), and resistance to extinction (Einon et al. 1975; Morgan et al. 1977). Rats isolated in this way also show an increased sensitivity to psychomotor stimulant drugs such as $d$-amphetamine and cocaine, both in terms of the locomotor enhancing properties of these agents and their ability to induce stereotyped behaviors (Sahakian et al. 1975; Jones et al. 1990, 1992), responses that depend on the mesolimbic and mesostriatal dopamine (DA) projections, respectively (Kelly et al. 1975). Consistent with these behavioral data are studies utilizing in vivo microdialysis techniques, which have provided evidence that isolation-reared rats exhibit greater elevations in extracellular DA in both dorsal and ventral striatum in response to systemic $d$-amphetamine (Jones et al. 1992), thereby 
substantiating the hypothesis that early social isolation produces enduring changes in central dopaminergic function.

Recently, there has been considerable interest in the modeling of aspects of the attentional deficits in schizophrenia by paradigms that can be used in both humans and experimental animals. In this regard, schizophrenic patients have been reported to show deficits in both prepulse inhibition (PPI) of the startle response, in which a weak lead stimulus markedly reduces the response to strong startle-eliciting stimuli (Ison and Hoffman 1983; Braff et al. 1992), and latent inhibition (LI), a procedure in which nonreinforced preexposure to a stimulus retards subsequent conditioning to that stimulus (Lubow 1973; Baruch et al. 1988). Both PPI and LI have been shown to be affected by treatments that alter central dopaminergic transmission. For example, PPI is disrupted by systemic administration of $d$-amphetamine or the direct DA agonist apomorphine, effects that can be blocked by neuroleptic drugs such as raclopride (Davis et al. 1990; Mansbach et al. 1988; Swerdlow et al. 1991). Similarly, the LI effect is abolished by systemic $d$-amphetamine, whereas neuroleptic drugs enhance the LI effect (Weiner et al. 1988; Weiner and Feldon, 1987). In the case of PPI, there is strong evidence that some of the effects of dopaminergic manipulations involve the ventral striatum and its associated neural circuitry. Thus, infusions of DA directly into the nucleus accumbens (NAC) have been shown to disrupt PPI (Swerdlow et al. 1990a): an effect that can be reversed by infusion of the gamma-aminobutyric acid (GABA)-ergic agonist muscimol into the ventral pallidum, a major target area of the NAC (Swerdlow et al. 1990b). The situation with LI is less clear, in that recent evidence has indicated that the disruption of LI induced by systemic $d$-amphetamine is unlikely to be due to DA-dependent mechanisms of the ventral striatum (Killcross and Robbins 1993). Furthermore, discrete lesions of the ventral subiculum, the source of a major connection between the hippocampus and the ventral striatum (Groenewegen et al. 1991) are without effect on LI (Clarke et al. 1992). It would seem that, although the LI effect is sensitive to treatments that perturb DA transmission, the precise neuroanatomic substrate for these effects remains unknown.

The changes in central DA function brought about by social isolation suggest that it may be of interest to compare the effects of this manipulation on PPI and LI. Given the basic finding that treatments that enhance dopaminergic transmission in the striatum reduce PPI, one main prediction would be that isolates should show similar deficits in PPI. In fact, previous experiments have indicated that early social isolation does indeed impair PPI (Geyer et al. 1993). Nevertheless, these data leave several questions unresolved: in particular, the developmental specificity of the effects of isolation. That is to say, does a period of isolation as an adult give rise to the same changes in PPI as that seen in animals isolated at the weaning age. This issue is important because both manipulations might be hypothesized to be forms of chronic mild stress, which is known to affect the functioning of the mesolimbicocortical dopaminergic systems (LeMoal and Simon 1991). It has rarely been considered, however, that stressors might affect these systems differentially depending on the age of the animal. Therefore, the first experiment compared the effects of isolation rearing (i.e., isolating weanlings) and isolation housing (i.e., isolating adults) on PPI of the acoustic startle response. The second experiment examined the effects of isolation rearing on the development of LI in a within-subject, conditioned-suppression paradigm. Here, any predictions must be less firm in that, as mentioned previously, the precise role of striatal DA mechanisms in the expression of LI is not settled. Accordingly, it could be predicted that social isolates would show no deficits in LI, with the caveat that isolation may modify LI by producing changes in DA function at sites other than the ventral striatum. In view of the particular uncertainty regarding the effects on LI of elevating DA transmission in ventral striatum, these animals were also subsequently assessed for levels of extracellular DA in the NAC utilizing in vivo microdialysis, a technique that provides a direct measure of presynaptic transmitter dynamics (Ungerstedt 1984).

\section{MATERIALS AND METHODS}

\section{Subjects and Housing Conditions}

The experiments used a total of 64 male, Lister Hooded rats, 40 weanlings ( 21 days postnatal) and 24 adults (weighing approximately $300 \mathrm{~g}$ ). All rats were obtained from Harlan Olac Ltd (Bicester, U.K.). On arrival at our laboratory, the animals were divided into two rearing conditions counterbalanced by weight. Animals were housed either in isolation or in social groups for the duration of the experiment. Social groups were housed in fours in cages $56 \times 38 \times 18 \mathrm{~cm}$, and isolated animals were housed singly in cages $45 \times 20 \times 20 \mathrm{~cm}$. All cages were of plastic construction with steel grid floors and underhanging sawdust trays (North Kent Plastic Cages Ltd, Dartford, U.K.). The animals were kept in a colony room maintained at $21^{\circ} \mathrm{C}$ on a 12-hour light/12-hour dark cycle (light on 0700) with free access to food and water. All rats could see, hear, and smell the other rats in the colony.

\section{Experimental Groups}

Testing began after 8 weeks of the various housing conditions. All animals were first scored for spontaneous 
activity in a novel environment. Thereafter, rats entered one of two experimental pathways. In the first experiment, a total of 48 animals were assessed for acoustic startle and PPI of startle. This group contained rats that had been isolated from weaning age as well as rats isolated as adults together with their respective social controls ( $n=12$ for each group). The former group was defined as isolation-reared animals and the latter as isolation-housed animals to distinguish between the two isolation conditions. In the second experimental pathway, 16 animals (eight isolation reared and eight social controls) were tested for the expression of LI in an on-baseline, within-subjects, conditioned-suppression paradigm followed by assessment of DA levels in the NAC using in vivo microdialysis techniques.

\section{Assessment of Spontaneous Activity}

Apparatus. Spontaneous locomotor activity was monitored in 16 individual wire-mesh photocell cages $(40 \times 25 \times 18 \mathrm{~cm})$. Each cage was fitted with two parallel horizontal infrared beams, $1 \mathrm{~cm}$ above the floor, spaced equally along the long axis of the cage. Interruption of either beam resulted in an incremental count for that cage. Beam breaks were the dependent measure and were recorded on-line in an adjacent room by a BBC Master Series microcomputer equipped with a SPIDER extension (Paul Fray Ltd, U.K.). Data from the 2-hour session were collected in 12, 10-min time bins.

Behavioral Procedures. All animals were naive to the activity boxes and were tested in batches of 16 between 1600 and 1800 hours, that is, towards the end of their light period.

\section{Assessment of Startle and Prepulse Inhibition of Startle}

Apparatus. A SR-LAB (San Diego Instruments, San Diego, CA) test station was used to detect startle responses. The illuminated, ventilated chamber contained a stabilimeter composed of an 8.2-cm diameter Plexiglas cylinder mounted on a Plexiglas base as described previously (Swerdlow et al. 1991). A speaker mounted $24 \mathrm{~cm}$ above the animal provided the background noise, prepulse stimuli, and startle stimuli, which were controlled by the SR-LAB. Startle responses were transduced by a piezoelectric accelerometer mounted below the cylinder, digitized (0-4095), rectified, and recorded as 1001 -millisecond readings, starting at the onset of each startle stimulus. The average of these 100 readings was used as the dependent measure.

Behavioral Procedures. For testing, rats were placed into the startle apparatus and allowed a 5-minute acclimation period during which a 65-dB [A] (all sound levels refer to the A scale) background noise was ad- ministered. The startle session began with three successive 120-dB startle stimuli of 30-millisecond duration, followed by five blocks of trials, each comprising two $120-\mathrm{dB}$ startle stimuli alone, four prepulse stimuli + startle stimuli, and two no-stimuli trials. Within each block, individual trials were distributed in a pseudorandom fashion. Four different intensities of acoustic prepulse stimuli $(2,4,8$, and $16 \mathrm{~dB}$ above background) were used, each prepulse being 30 millisecond in duration. The time from onset of the prepulse to onset of the startle stimulus was always 100 milliseconds. In all experiments, the variable interval between trials averaged 10 seconds; hence, the session lasted approximately 15 minutes.

\section{Assessment of Latent Inhibition in a Within-Subjects Conditioned-Suppression Paradigm}

Apparatus. Four standard operant chambers $(26.5 \times$ $22 \times 20 \mathrm{~cm}$; Campden Instruments) housed in lightand sound-attenuating boxes were used for the procedures. The chambers were illuminated by a diffuse light source located above the translucent plastic ceiling. The floor of the chambers consisted of 16, 5-mm diameter, steel rods spaced $5 \mathrm{~mm}$ apart. These grids were each connected to a shock generator (Model 512/C; Campden Instruments), and a shock scrambler (Model 512/S; Campden Instruments), set at $0.3 \mathrm{~mA}$. Two discrete auditory stimuli were available in each chamber: one was a 3-kHz tone, produced by a Sonalert module (Model SC 628) and delivered through a wall-mounted speaker located opposite to the food magazine, and the other derived from a heavy-duty relay mounted behind the back wall providing a $10-\mathrm{Hz}$ clicker. Each chamber contained a single lever, $3.8-\mathrm{cm}$ wide, located to the lefthand side of a central, recessed magazine that provided access, via a hinged Plexiglas panel, to a dipper delivering $20 \%$ sucrose solution. Lever presses were the dependent measure (see below). A BBC Master-128 microcomputer, equipped with a SPIDER extension for on-line control (Paul Fray Ltd., UK), controlled the equipment and recorded the data.

Behavioral Procedures. Two days before the beginning of the test procedures, the animals were placed on a 22.5-hour schedule of food deprivation and maintained on this schedule throughout the rest of the experiment. Water was available ad libitum. All animals received two initial 30-minute magazine-training sessions with the levers removed from the chambers; sucrose was delivered according to a variable time 60 second(VT60) schedule. The levers were then replaced and two 30-minute sessions given on a continuous reinforcement schedule in which every lever press was rewarded, up to a maximum of 60 rewards per session. For the next two sessions, a variable interval 30 -second 
(VI30) schedule was employed: the first session lasting 30 minutes and the second 40 minutes. Thereafter $40-$ minute sessions on VI60 were used. After three such 40-minute VI60 sessions had been given, the preexposure phase of the experiment began.

Isolated and social animals were divided randomly into two groups. One group in each of the two conditions was assigned to preexposure to the $3-\mathrm{kHz}$ tone and the other to preexposure to the $10-\mathrm{Hz}$ clicker $(n=$ 4 in all cases). Preexposure consisted of three 40-minute on-baseline VI60 lever-press sessions during which the to-be-conditioned stimulus was presented 12 times according to a pseudo-random schedule. Each presentation lasted 30 seconds, and the average ISI was approximately 3 minutes. The preexposure sessions and all subsequent sessions were conducted on alternate days. On the intervening days, animals were given one, 30minute VI60 lever-press session to maintain baseline responding. Following the preexposuresessions, an assessment of unconditioned suppression to the nonpreexposed stimulus was made. This session served to remove the initial disruption of lever pressing observed following presentation of any novel stimulus (Baker and Mercier 1982) and also provided a demonstration that the animals could, in fact, distinguish between the two auditory stimuli. During a VI60 lever-press session, both auditory stimuli were presented twice to every animal, in a counterbalanced order, and at equal intervals throughout the session. Finally, conditioning to both of the auditory stimuli occurred over four subsequent conditioning sessions. In each of these sessions, each of the auditory stimuli was presented once, again in a counterbalanced order, but in this case, the final 0.5 seconds of the stimulus presentation was accompanied by a $0.3-\mathrm{mA}$ electric shock, delivered through the grid floor of the cage. The first conditioned stimulus was presented 10 minutes after the start of the session, and the second conditioned stimulus came 23 minutes later. This interval provided a sufficiently long period between the two shock presentations to allow for recovery from any suppression of baseline responding following shock presentation.

\section{Measurement of Extracellular Dopamine in Nucleus Accumbens}

Apparatus. Basal and $d$-amphetamine stimulated levels of extracellular DA in the nucleus accumbens were assessed using in vivo microdialysis. Animals were anesthetized with halothane $\left(0.9 \%\right.$ in $\left.\mathrm{O}_{2}\right)$ and placed in a stereotactic frame with the incisor bar set at $+5 \mathrm{~mm}$. The skull was exposed, a burr hole made, and a 2-mm dialysis probe lowered into the brain so that the tip of the probe was at the following stereotactic coordinates, AP: $+3.4 \mathrm{~mm}$, L: $-1.5 \mathrm{~mm}$ (from bregma), V: $-7.5 \mathrm{~mm}$ (from dura). The dialysis probe was of a concentric de- sign, made of 23-gauge steel tubing coupled to Hospal dialysis membrane (nominal 5000 molecular weight cut off). The probe was perfused with ACSF at a flow rate of $2 \mu \mathrm{l} / \mathrm{min}$. The composition of the ACSF was as follows: $\mathrm{NaCl} 120 \mathrm{mmol} / \mathrm{L}, \mathrm{NaHCO}_{3} 27 \mathrm{mmol} / \mathrm{L}, \mathrm{KCl} 2.5$ $\mathrm{mmol} / \mathrm{L}, \quad \mathrm{NaH}_{2} \mathrm{PO}_{4} \quad 0.6 \mathrm{mmol} / \mathrm{L}, \quad \mathrm{Na}_{2} \mathrm{HPO}_{4} \quad 1.27$ $\mathrm{mmol} / \mathrm{L}, \mathrm{Na}_{2} \mathrm{SO}_{4} 1 \mathrm{mmol} / \mathrm{L}, \mathrm{MgCl}_{2} 0.47 \mathrm{mmol} / \mathrm{L}, \mathrm{CaCl}_{2}$ $1.5 \mathrm{mmol} / \mathrm{L}, \mathrm{pH} 7.4$. At room temperature, the in vitro recovery of the probe for DA was $12 \%$ to $14 \%$. The dialysis data were not corrected for recovery. Dialysates were collected every 30 minutes and immediately injected into a microbore high-performance liquid chromatography (HPLC) system. The HPLC system consisted of a BAS PM-60 pump connected via a column splitter to a reverse-phase analytical column (Spherisorb 30DS2; $10 \mathrm{~cm} \times 1 \mathrm{~mm}$ inner diameter). The mobile phase, flowing at $70 \mu \mathrm{l} / \mathrm{min}$, was composed of 152 $\mathrm{mmol} / \mathrm{L}$ citric acid, $15 \mathrm{mmol} / \mathrm{L}$ sodium acetate, $1 \mathrm{mmol} / \mathrm{L}$ octyl sulphate, $0.8 \mathrm{mmol} / \mathrm{L}$ ethylenediaminetetraacetic acid and $8 \%$ methanol, pH 3.6. Separated compounds were detected using a BAS LC-4B cell, fitted with a glassy carbon electrode held at a potential of $+0.7 \mathrm{~V}$ relative to $\mathrm{Ag} / \mathrm{AgCl}$ reference electrode. The output from the detector was analyzed by an integrator. Identification and quantification of the DA peak was achieved by comparison with external standards of known concentration. The identity of the DA peak was further confirmed by spiking with an internal standard. Under the conditions described the maximum sensitivity for DA was $0.25 \mathrm{pg}$ (signal-to-noise ratio of 3 ).

\section{Dialysis Procedures}

All the dialysis experiments followed the same pattern. After a 1-hour postimplantation period, 3 hours of baseline determinations were taken followed by a further 3 hours of collection after the administration of $d$-amphetamine (free base, $2 \mathrm{mg} / \mathrm{kg}$ in $0.5 \mathrm{ml}$ saline IP). Under the general conditions described, both basal and stimulus-evoked $\left(75 \mathrm{mmol} / \mathrm{L} \mathrm{K}^{+}\right)$release of DA were calcium dependent $(60 \%$ reduction in basal levels, $75 \%$ reduction in stimulus-evoked levels; data not shown). Throughout the experiment, the core temperature of the animal was monitored with a rectal probe and maintained at $37^{\circ} \mathrm{C}$.

\section{Histologic Verification of Probe Placements}

Immediately after the final dialysis sample, animals were killed by intraperitoneal injection of pentobarbital and perfused transcardially with phosphate buffered

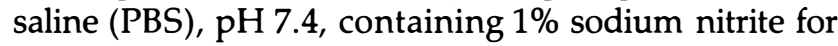
2 minutes, followed by $250 \mathrm{ml}$ of $4 \%$ paraformaldehyde in PBS containing $0.4 \%$ picric acid. The brains were removed from the skull, postfixed in the same solution for 1 hour, and transferred to $25 \%$ sucrose in Tris- 
buffered saline. After equilibration in the sucrose, sections were cut on a freezing stage sledge at $4^{\circ} \mathrm{C}$ prior to staining. One in five series was mounted on gelatincoated slides and stained for cresyl violet and acetylcholinesterase, respectively. After staining, slides were dehydrated in alcohols, cleared in xylene, and mounted in DPX.

\section{Statistical Analyses}

Spontaneous Activity Scores. The activity data (beam breaks) were analyzed using a three-way analysis of variance (ANOVA), with two between-subjects factors, Social Experience (i.e., social or isolate) and Age (i.e., weanling or adult when treatment commenced), and one repeated within-subjects factor, Time Bin.

Startle and Prepulse Inhibition of Startle. Startle responses were analyzed using two- and three-way ANOVAs, with two between-subjects factors, Social Experience and Age, and one within-subjects factor, Block. Prepulse inhibition was defıned as the difference between the responses on the $120-\mathrm{dB}$ pulse-alone trials and the responses on the prepulse $+120-\mathrm{dB}$ pulse trials divided by the average response on the corresponding $120-\mathrm{dB}$ pulse-alone trials multiplied by 100 . Prepulse inhibition data for each prepulse-type (i.e., 2, 4, 8, or $16 \mathrm{~dB}$ above background) were analyzed using two- and three-way ANOVAs with two between-subjects factors, Social Experience and Age, and one within-subjects factor, Block. Where appropriate, post hoc specific comparisons were made using Duncans Multiple Range Test (Winer, 1971).

Latent Inhibition. Because the experiments presented here used a within-subjects design, the analyses were performed on the number of lever presses made during the conditioned stimulus. The standard measure of conditioned suppression, namely a suppression ratio, is designed to reduce between-subject variance. Because the comparison of major interest in the present study is a within-subjects comparison and there were no differences in baseline rates of lever pressing between isolation- and socially reared groups in any of the experiments (see Results), use of suppression ratios would have been inappropriate and unnecessary. The variance of the data was found to increase with the mean. Accordingly, all lever press data presented were subjected to a square-root transformation prior to twoand three-way ANOVAs, with one between-subjects factor, Social Experience, and three within-subjects factors, Preexposure, Trial, and Response Bin.

Dialysis Data. Extracellular levels of DA were expressed in picomoles per $60 \mu \mathrm{l}$ of dialysate and were not corrected for probe recovery. The data were analyzed using a two-way ANOVA with one between- subjects factor, Social Experience, and one withinsubjects factor, Time Bin. To meet the assumptions of equal variance and normality, basal and drug response data were analyzed separately and log transformed. Where appropriate, post hoc specific comparisons were made using Duncans Multiple Range Test(Winer, 1971).

\section{RESULTS}

\section{Spontaneous Activity in Novel Environment}

Figure 1 shows the activity scores in 10-minute time bins for isolation-reared and isolation-housed animals, together with their respective social controls on exposure to the activity cages. All four groups showed evidence of habituation over the 2-hour test period, as indicated by a significant main effect of Time Bin $(F[11,660]=$ $80.41, p<.0001)$ in the three-way repeated-measures ANOVA with factors Social Experience, Age, and Time $B i n$. The ANOVA also revealed a significant main effect of Social Experience $(F[1,60]=19.89, p<.0001)$, reflecting increased activity in isolated animals. This effect was independent of the age of the animal when isolated; there was no significant Age by Social Experience interaction and was not related to any group differences in the rate of change of activity over the test period;

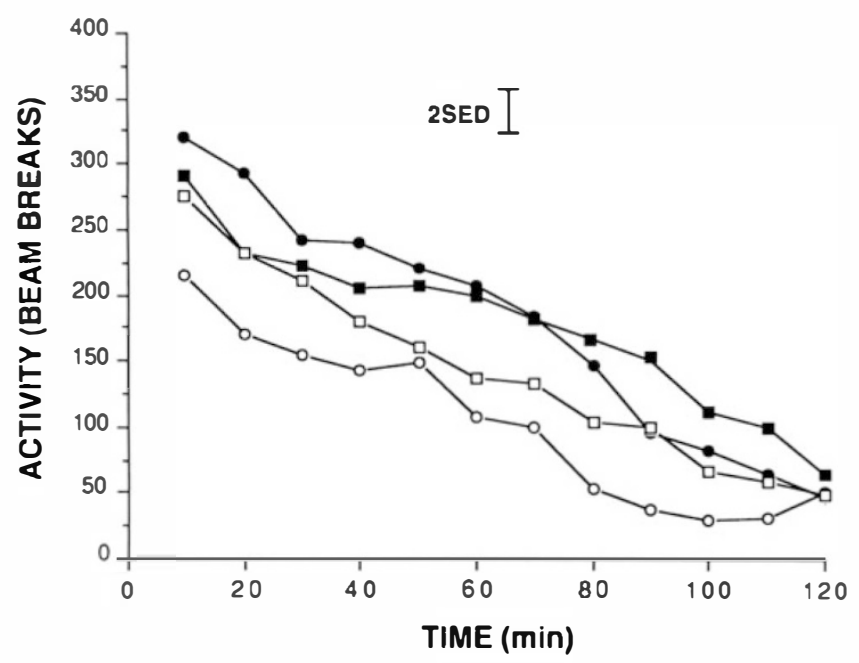

Figure 1. Time course of the locomotor activity of isolationreared ( $\square$ ) and isolation-housed (O) animals together with their social control groups, socially reared $(\square)$ and socially housed (O). The data are expressed as the number of beam breaks per 10 -minute bin $(n=12)$. The error bar represents twice the standard error of the difference between means (SED) for the three-way interaction with factors Social Experience, Age, and Time Bin. The SED is the denominator for making pairwise comparisons post hoc. Hence, the error bar provides an appropriate means of visually evaluating significance between pairs of points. The relevant formulae are given in Cochran and Cox (1957). 

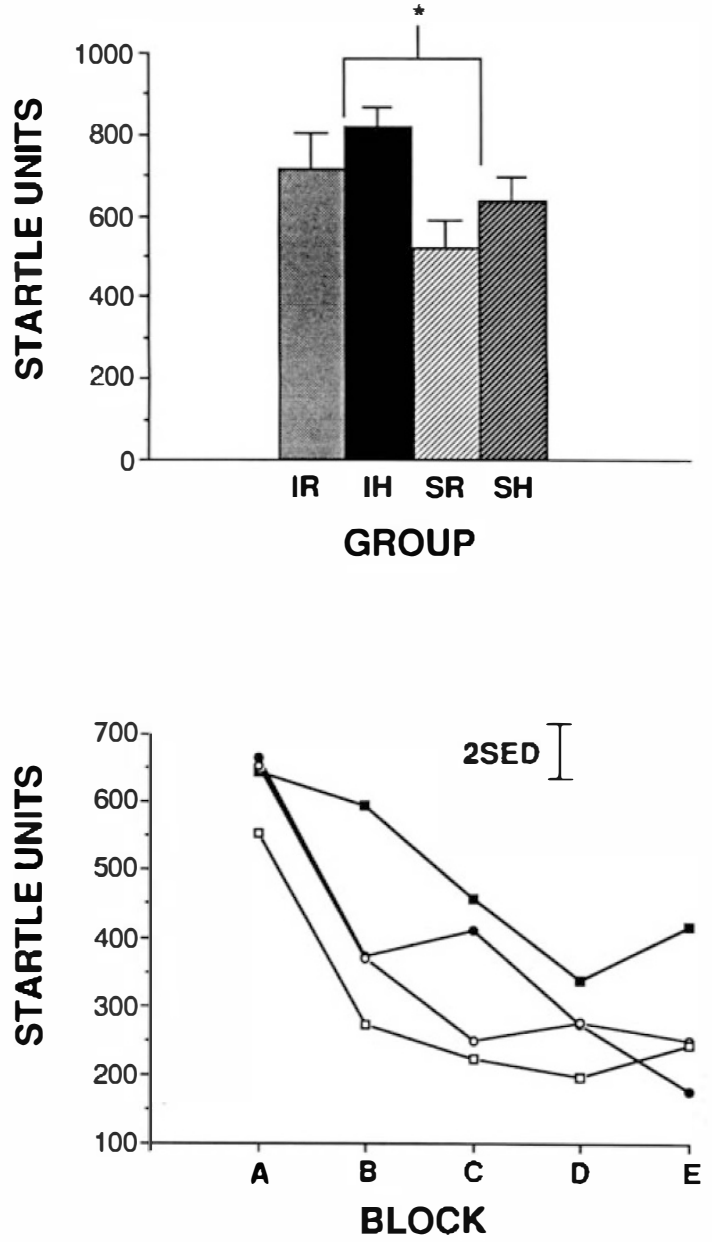

Figure 2. A: Startle responses for the initial three successive, $120-\mathrm{dB}$ startle stimuli of the startle session as exhibited by isolation-reared (IR), isolation-housed (IH), socially reared $(\mathrm{SR})$, and socially housed $(\mathrm{SH})$ animals. The data are expressed in arbitrary startle units and are the mean plus or minus standard error of the mean (SEM) $(n=12)$. * Denotes a significant difference $(p<.05)$ between the mean values obtained for isolates (both isolation reared and isolation housed) and social controls (Duncans Multiple Range Test). B: Mean startle responses to the $120-\mathrm{dB}$ startle stimuli measured over the five blocks of trials comprising the remainder of the startle session and from which the extent of any PPI was assessed. The key to experimental groups is the same as in Figure 1. The bar represents twice the SED between means for the threeway interaction with factors Social Experience, Age, and Block.

there was no signifıcant interaction involving Time Bin. Therefore, social isolation for a period of 8 weeks, in both the weanling and the adult rats, led to increased activity in the novel surroundings of the activity cages.

\section{Startle and Prepulse Inhibition of Startle}

Startle. Figure 2A illustrates acousticstartle responses for isolation-reared and isolation-housed animals, to- gether with their respective social controls, for the first three presentations of the $120-\mathrm{dB}$ startle stimulus. The two-way ANOVA of these data with factors Social Experience and Age revealed a significant main effect of Social Experience $(F[1,44]=5.51, p<.05)$ and no interaction, reflecting the fact that isolated animals (both isolation reared and isolation housed) were hyperreactive to these initial stimuli. Specific post hoc comparisons confirmed this conclusion. By contrast, there were no significant between-group differences in the subsequent responses to the $120-\mathrm{dB}$ stimuli used to assess PPI (see Fig. 2B). Clearly, however, there was some degree of habituation on repeated presentation of the startle stimulus in all groups. This habituation was confirmed by a main effect of Block $(F[4,176]=20.37, p<.0001)$ in the three-way ANOVA with factors Social Experience, Age, and Block. No-stimulus values, which averaged approximately 8 startle units, did not differ over Block or between groups.

Prepulse Inhibition of Startle. Because the 2- $\mathrm{dB}$ and 4- $\mathrm{dB}$ prepulse stimuli failed to alter startle responses in all experimental groups, these data are not shown. Figure 3A illustrates the effects of the 8-dB prepulse stimulus on startle response. It is important to note that the data are collapsed across blocks of trials. This maneuver was justified because the three-way ANOVA with factors Social Experience, Age, and Block failed to detect any effects of, or interactions with Block. That is, for all groups, prepulse effects were remaining constant over the session. Hence, any between-group differences were not due to differential rates of habituation of the startle response itself. From Figure 3A, it can be seen that in three of the experimental groups, the 8-dB prepulse reduced startle responses by some $50 \%$, a typical PPI effect. By contrast, in the isolationreared group, this prepulse essentially failed to modify responding to the startle stimulus. The two-way ANOVA, with factors Social Experience and Age, revealed a significant Social Experience by Age interaction $(F[1,44]=6.44, p<.002)$. Specific post hoc comparisons indicated that the isolation-reared group was significantly different from the other three groups, confirming the above conclusions. The effects of the 16$\mathrm{dB}$ prepulse stimulus on acoustic startle are shown in Figure 3B. This larger prepulse stimulus increased the degree of PPI in all experimental groups, although again, the absolute extent of PPI appeared to be less in the isolation-reared group. Nevertheless, increasing the intensity of the prepulse stimulus from $8 \mathrm{~dB}$ to 16 $\mathrm{dB}$ abolished the significant difference between groups, possibly due to a ceiling effect. Together, these data are consistent with the following conclusions: PPI of acoustic startle was selectively reduced in animals isolated at weaning age; the effect was not dependent on underlying changes in startle itself; and given the ability of more intense prepulse stimuli to elicit inhibition in 

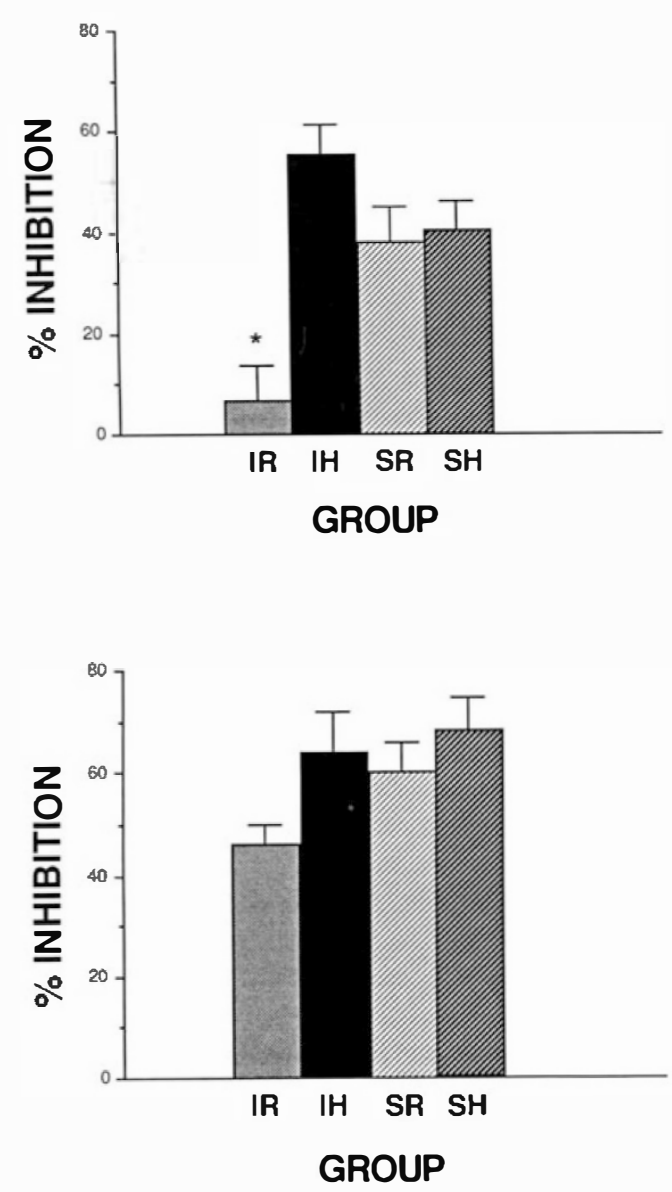

Figure 3. A: The percentage inhibition of startle produced by a prepulse stimulus $8 \mathrm{~dB}$ above the $65-\mathrm{dB}$ background presented 100 milliseconds prior to the $120-\mathrm{dB}$ startle stimuli, meaned over five blocks of trials. The key to experimental groups is the same as in Figure 2A. * Denotes a significant difference $(p<.05)$ between isolation-reared animals and the other three experimental groups (Duncans Multiple Range Test). B: Percentage inhibition of startle produced by a larger prepulse stimulus, $16 \mathrm{~dB}$ above background. In both cases, the data are the mean $\pm \operatorname{SEM}(n=12)$.

this group (albeit less than that seen in other experimental groups), it was specifically the threshold for inhibition that seemed to have been altered.

\section{Latent Inhibition in a Conditioned-Suppression Paradigm}

Baseline Rates. One animal from the tone preexposed isolation-reared group and one from the tone preexposed socially reared group failed to respond following the first shock presentation; thus, they were dropped from the study and all subsequent analyses. Baseline rates of lever pressing in the isolate and social groups were not significantly different. The mean rates of lever pressing during the 30-second period immediately prior to the conditioned-stimulus onsets (preconditioned-stimulus period) across all conditioning trials for isolation-reared and socially reared controls were: Isolates $=7.8$; Socials $=5.7$. A two-way ANOVA with Social Experience and Preexposure did not reveal any significant main effects or any interaction.

Unconditioned Suppression. Figure 4A shows the mean number of lever presses made during presentation of the preexposed and nonpreexposed stimuli from the two unconditioned stimulus exposures and from the first conditioning trial. Clearly, both groups of animals could discriminate between the two stimuli and demonstrated a significant degree of unconditioned suppression to the novel stimulus. This unconditioned suppression habituated at the same rate in both the isolated and social groups, and there was no difference in the suppression of lever pressing caused by either stimulus in either group by the first conditioning trial (see Fig. 4B). These conclusions are supported by the three-way ANOVA with Social Experience, Preexposure, and Trial that showed no significant main effect of Social Experience or interaction involving this factor. There were, however, significant main effects of Trial $(F[2,24]=12.224, p<.001)$ and Preexposure $(F[1,12]=20.945, p<.001)$, together with a significant Trial by Preexposure interaction $(F[2,24]=8.563, p<$ .005). Analysis of Simple Effects from this interaction indicated a significant effect of Trial on the preexposed stimulus $(F[2,24]=11.814, p<.001)$ and a significant effect of preexposure on the first unconditioned presentation $(F[1,12]=23.697, p<.001)$ but not on the second or third presentation. It would seem, therefore, that lever pressing was significantly suppressed on the initial presentation of the nonpreexposed stimulus, but that this suppression had habituated by the first conditioning trial.

Conditioned Suppression. Figure 4B shows the mean number of lever presses made by isolates and socials during presentation of the preexposed and nonpreexposed stimuli for the four conditioning trials. As expected, socials showed a strong LI effect manifest as a slowed conditioning to the preexposed stimulus relative to the nonpreexposed stimulus. Isolates showed qualitatively similar effects but the absolute values for lever pressing were higher during both preexposed and nonpreexposed stimuli. Thus, although there was no evidence of any reduction in LI in isolates, it would appear that lever pressing was influenced by some type of generalized response disinhibition in this group. These conclusions were supported by the three-way ANOVA with Social Experience, Preexposure, and Trial, which revealed no main effect of Social Experience but significant main effects of Preexposure $(F[1,12]=$ $13.233, p<.005)$ and Trial $(F[3,36]=10.866, p<.001)$. None of the interactions were significant.

Shock-Induced Suppression. Figure 4C shows the distribution of lever press responding for isolates and so- 

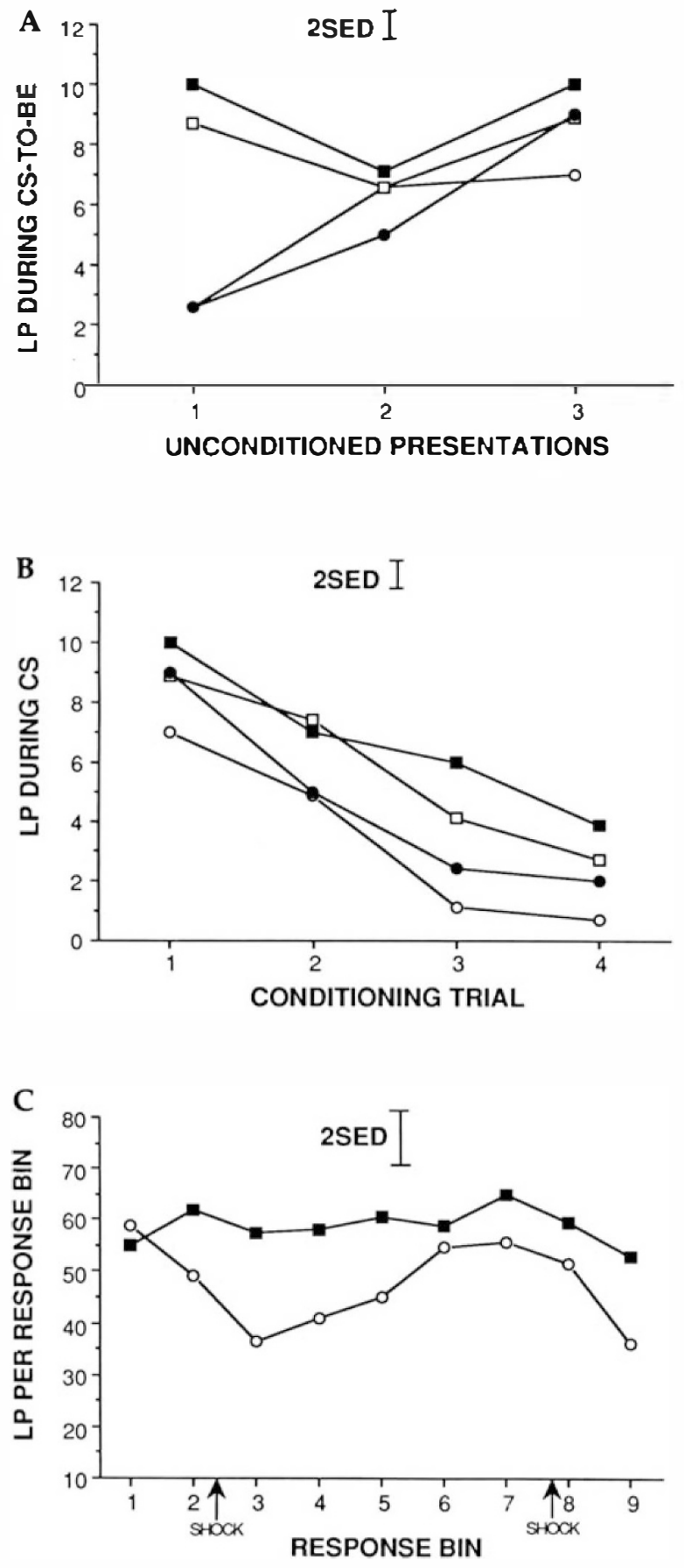

Figure 4. A: Mean number of lever presses made during presentations of preexposed and nonpreexposed stimuli prior to conditioning. Closed symbols refer to isolation-reared animals, open symbols to socially reared animals. Circles show responding to the nonpreexposed stimulus, squares to the preexposed stimulus. $n=4$ in each cell. The bar represents twice the SED between means for the three-way interaction with factors Social Experience, Preexposure, and Trial. B: Mean number of lever press made during presentations of preexposed and nonpreexposed stimuli during conditioning. Note the appearance of LI (retarded conditioning to the preexposed stimulus) in both groups and the general retardation of conditioning in isolation-reared animals. Again, the bar cials meaned across the four conditioning sessions. It can be seen that there was a reduced degree of suppression postshock in animals reared in isolation. This impression was supported statistically. The three-way ANOVA with Social Experience, Response Bin, and Trial revealed a significant main effect of Response Bin $(F[8,96]=3.979, p<.001)$ and a significant Social Experience times Response Bin interaction $(F[8,96]=$ $2.186, p<.05)$. Analysis of Simple Effects on this interaction revealed that although there was a significant effect of Response Bin in control animals $(F[8,96]=$ $5.229, p<.001)$, there was no such effect in the isolationreared animals $(F[8,96]<1, N S)$. It would seem then, that the elevated rates of responding manifest by isolates in the conditioning stimuli were probably the result of a reduced impact of the shock in this group; the critical point being that such an effect would generalize across preexposed and nonpreexposed stimuli, precisely what was observed.

\section{Extracellular Levels of Dopamine in Nucleus Accumbens}

Figure 5 shows the basal and $d$-amphetamine stimulated levels of extracellular DA in the NACs of those animals assessed for LI, as measured by in vivo dialysis. All dialysis probes were placed accurately within the medial portion of the nucleus (see Fig. 6) with an anteroposterior range of less than $1 \mathrm{~mm}$. In both social and isolation-reared animals, basal levels of DA were within the range of values reported by others (Zetterstrom et al. 1983). A two-way ANOVA involving Social Experience and Time Bin failed to reveal any significant effects of either of these factors on basal levels of DA. By contrast, main effects of Time Bin $(F[8,96]=$ $12.449, p<.0001)$ and Social Experience $(F[1,12]=$ 23.317, $p<.0005)$, together with a significant Social Experience by Time Bin interaction $(F[8,96]=5.418, p<$ $.0001)$, were present in the postamphetamine data. That is to say, although there were no differences in basal levels of DA, the administration of $d$-amphetamine had a much greater effect in enhancing DA levels in the isolation-reared animals than in the socially reared con-

represents twice the SED between means for the three-way interaction with factors Social Experience, Preexposure, and Trial. C: Pattern of lever press responding across conditioning sessions presented as the mean number of presses made during nine, equally sized time bins across the 40-min session. Arrows indicate the points of postconditioned stimulus (CS) shock presentation (after 10 minutes and 33 minutes). Note the reduceddegree of suppression in the isolation-reared animals $(\boldsymbol{\square})$ compared to socially reared controls $(\mathrm{O})$. The bar represents twice the SED between the means for the threeway interaction with factors Social Experience, Response Bin, and Trial. 


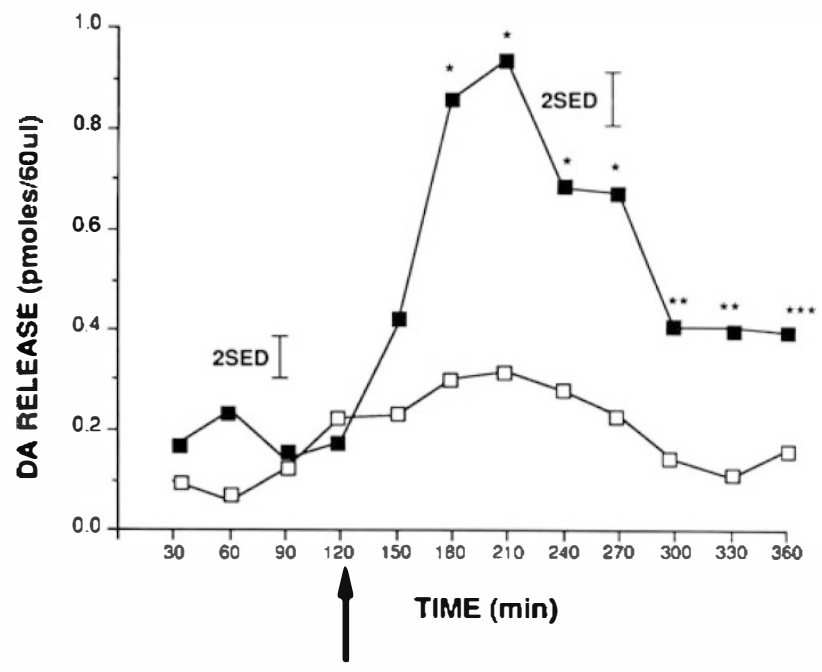

Figure 5. Levels of extracellular DA in dialysates obtained from NACs of isolation-reared ( $\square$ ) and socially reared ( $\square$ ) animals under basal and $d$-amphetamine stimulated conditions. The animals were anesthetised with $0.9 \%$ halothane in $\mathrm{O}_{2}$. $d$-Amphetamine (free base, $2 \mathrm{mg} / \mathrm{kg}$ ) was administered intraperitoneally at the time point shown by the arrow. The data are expressed as picomoles per $60 \mu \mathrm{I}$ dialysate and are notcorrected for probe recovery. Basal and postamphetamine data were analyzed separately. In each case, the bars represent twice the SED between means for the two-way interaction with factors Social Experience and Time Bin. * $(p<.001)$, ${ }^{\star \star}(p<.02)$, and ${ }^{\star \star \star}(p<.05)$ denote significant differences between isolation-reared and socially reared animals (analysis of Simple Effects from the significant interaction between factors Social Experience and Time Bin).

trols. Furthermore, there were additional group differences manifest over time, in that DA levels returned to basal levels 3 hours postamphetamine in the socials, whereas levels were still elevated in the isolates. Specific post hoc comparisons confirmed these conclusions.

\section{DISCUSSION}

The main finding of the experiments was that social isolation from the weaning age in rats disrupted performance in only one of the two attentional paradigms that have been suggested as animal models related to schizophrenia. This treatment had no effect on LI but confirming previous observations (Geyer et al. 1993), reduced PPI of the acoustic startle response. Furthermore, there was evidence that the effects of social isolation on PPI were developmentally specific in that social isolation in adult rats was without effect on PPI.

The disruption of PPI following isolation rearing is consistent with the neurochemical changes in DA function produced by this manipulation, namely, enhanced effects of systemic $d$-amphetamine challenge on DA release in the ventral striatum. Both the indirectly acting agonist $d$-amphetamine and direct agonists, such

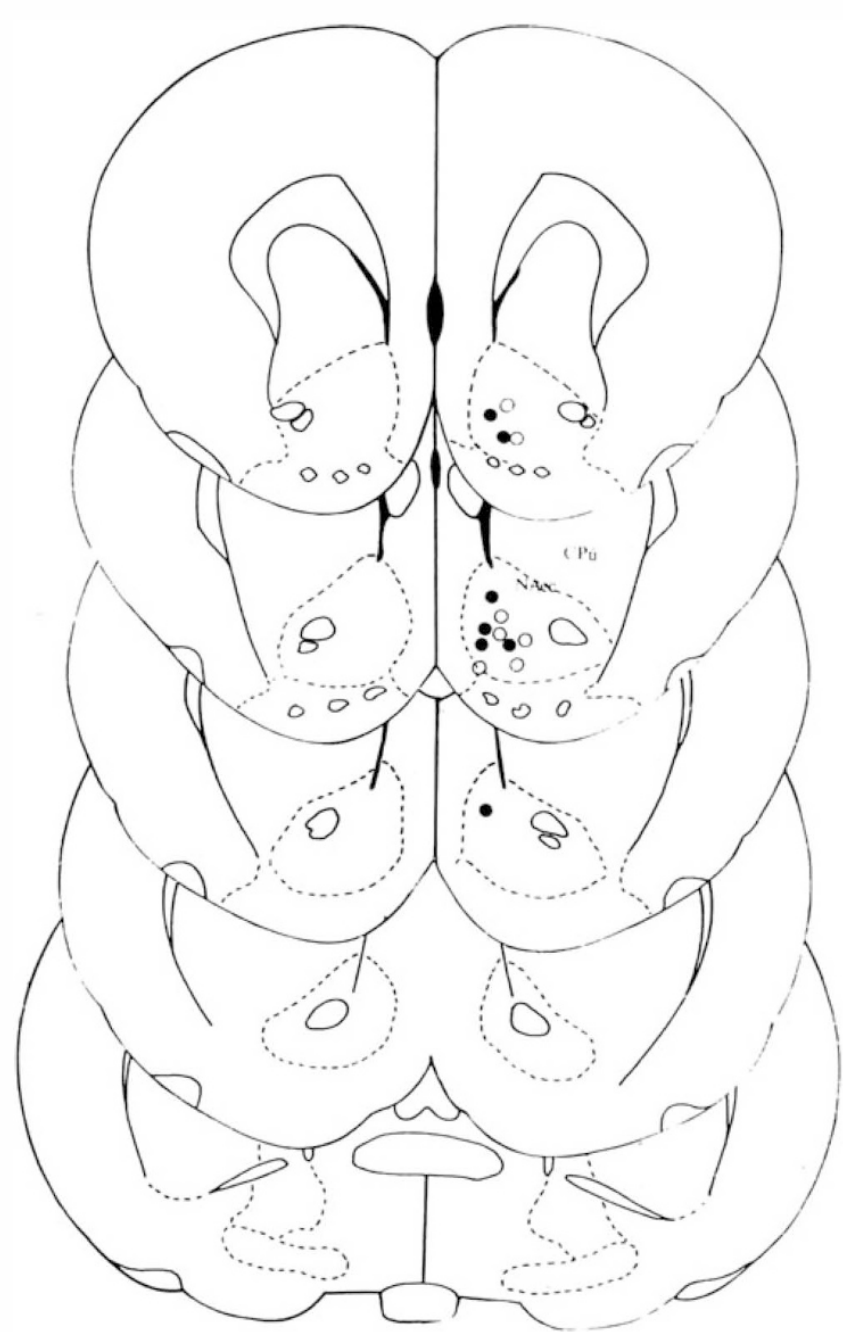

Figure 6. Distribution of dialysis probe placements within the ventral striatum. Sites were verified from sections stained with cresyl violet that were examined under the microscope. Isolation reared animals (O), socially reared animals (O). $\mathrm{NAcc}$-nucleus accumbens, $\mathrm{CPu}$-caudate putamen.

as apomorphine or quinpirole, have been shown to disrupt PPI, effects that can be blocked by $\mathrm{D}_{2}$-receptor antagonists (Geyer et al. 1990; Swerdlow et al. 1991). Given the additional evidence that the disruption of PPI by isolation rearing can be reversed by treatment with nonsedative doses of the selective, $D_{2}$ antagonist raclopride (Geyer et al. 1993), it would seem probable that the perturbations in DA transmission seen in animals reared in isolation are involved, to some extent, in the impairments seen in PPI.

By contrast, the data provide no support for the involvement of ventral striatal dopaminergic mechanisms in the expression of LI. Although published results have suggested an effect of dopaminergic drugs on LI (Weiner et al. 1988; Killcross and Robbins 1993; Killcross et al. in press), these findings are not inconsistent with the present lack of effect of isolation rearing. A recentstudy has shown that behaviorally active doses 
of $d$-amphetamine infused directly into the ventral striatum have no effect on LI in the same within-subjects conditioned-suppression LI procedure as used here (Killcross and Robbins 1993). Therefore, the lack of effect of isolation rearing might be expected, even though this treatment evidently leads to increases in DA activity in the ventral striatum. Presumably, the disruptive effects of systemic $d$-amphetamine on LI that we have also observed (Killcross and Robbins, 1993) are occurring at a site or sites other than the ventral striatum.

Although not affecting LI, isolation rearing decreased the suppression of lever pressing observed in the presence of aversive conditioned stimuli, suggesting impaired mechanisms of behavioral inhibition. This finding is worthy of note as the disinhibition produced by isolation rearing was paralleled by intraaccumbens $d$-amphetamine (Killcross and Robbins, 1993) and lesions of the ventral subiculum (Clarke et al. 1992), both of which failed to impair LI. This parallel suggests a role for altered mesolimbic DA in the behavioral disinhibition exhibited by isolates, both here, and in other situations (Morgan et al. 1977).

The effects of isolation rearing on PPI were specific in several ways. First, they were independent of any underlying baseline changes in the startle response itself, as the reduction in PPI was present at all stages of habituation to the startle stimulus. Second, the rightward shift in responsivity to the prepulse stimuli, suggestive of an elevated threshold for PPI in isolationreared animals, was probably not due to basic sensory loss, because the response to the $120-\mathrm{dB}$ startle stimulus was unaffected, whereas it would be expected to be reduced in rats with hearing impairments. Third, although isolation rearing increases general locomotor activity, this behavior cannot account for the reductions in PPI because activity within the startle apparatus (as indicated by the No-Stimulus values) was not affected by isolation, and rats isolated as adults, which were also hyperactive, did not show deficits in PPI.

The sparing of PPI in animals isolated as adults is of particular interest because it occurred, as noted above, in parallel with increases in both initial startle reactivity and spontaneous locomotor activity that were common to both isolated groups. It seems unlikely, therefore, that the differential effect of the two isolation conditions on PPI was due to the fact that 8 weeks of isolation has quantitatively less impact as a stressor when experienced as an adult. Furthermore, adults isolated for periods of up to 24 weeks continue to show normal PPI (our unpublished data). Rather, the behavioral dissociations suggest that the changes in PPI, startle, and locomotor activity were not redundant measures of the same effect of social isolation and that in young animals, this treatment exerts a qualitatively different effect on brain function than in older animals, perhaps as a result of differences in plasticity between the developing and mature brain. With regard to the impact of isolation housing on DA function in ventral striatum, it would be of clear interest in future studies to conduct dialysis experiments on animals isolated as adults. Such studies would aid in the interpretation of the behavioral differences between isolation-reared and isolation-housed subjects in terms of neural substrates.

The differential effects of isolation rearing on PPI and LI also provide strong evidence that these two phenomena do in fact represent the expression of two quite different psychological functions. Although they have both been considered to reflect attentional mechanisms (Weiner 1990; Braff and Geyer 1990), it is clear that they are psychologically and neurally distinct. Indeed, recent analyses have considered the possibility that $\mathrm{LI}$ is a function of associative rather than attentional processes (Killcross et al. in press), whereas PPI has been attributed to a hypothetical "sensory gating" system (Braff and Geyer, 1990). Within this theoretical framework, common deficits in PPI in schizophrenia and in the rat reared in social isolation, perhaps related to ventral striatal dysfunction, could be seen as impairments in sensory filtering of the prepulse stimulus. Alternatively, DA-dependent ventral striatal outflow may exert descending influences on the expression and modulation of sensorimotor reflexes at the brainstem level. According to this view, neurochemical changes in the ventral striatum produced, for example, by dopaminergic drugs or as in the present case, by isolation rearing, occasion changes in state that indirectly affect the processing of prepulse stimuli, rather than modifying perception of the stimuli per se.

In summary, the present investigation directly compared two putative models of the attentional deficits seen in schizophrenia. The results indicate a developmentally specific effect of social isolation on PPI of the acoustic startle response but no effects of isolation rearing on LI. The use of social isolation in the weanling rat is particularly relevant given that it provides a nonpharmacologic, nonlesion means of perturbing ventral striatal DA function thought to be important in the neuropathology of schizophrenia. Further studies will be required to characterize possible neurochemical changes underlying the other aspects of the isolation syndrome described here. In this regard, isolationinduced alterations in serotonin transmission might be of particular significance (Jones et al. 1992).

\section{ACKNOWLEDGMENTS}

This research was supported by a Pilot Project grant from the John D. and Catherine T. MacArthur Foundation Mental Health Network I: The Psychobiology of Depression and Other Affective Disorders and, in part, by a Wellcome Trust Programme grant. Mark A. Geyer was in receipt of a Fogarty Senior International Fellowship. A.S. Killcross was supported 
by a studentship from the Science and Engineering Council (U.K.) and F.S. Hall by an individual predoctoral fellowship (DA 05408) from the NationalInstitute on Drug Abuse (USA).

\section{REFERENCES}

Baker AG, Mercier P (1982): Extinction of context and latent inhibition. Learn Motiv 13:391-416

Baruch I, Hemsley D, Gray JA (1988): Differential performance of acute and chronic schizophrenics in a latent inhibition task. J Nerv Ment Dis 176:598-606

Braff DL, Geyer MA (1990): Sensorimotor gating and schizophrenia. Arch Gen Psychiatry 47:181-188

Braff DL, Grillon C, Geyer MA (1992): Gating and habituation of the startle reflex in schizophrenic patients. Arch Gen Psychiatry 49:206-215

Clarke AJM, Feldon J, Rawlins JNP (1992): Aspiration lesions of rat ventral hippocampus disinhibit responding in conditioned suppression or extinction, but spare latent inhibition and the partial reinforcement extinction effect. Neuroscience 48:821-829

Cochran WG, Cox GM (1957): Experimental Designs. 2nd Ed. New York, Wiley

Davis M, Mansbach RS, Swerdlow NR, Campeau S, Braff DL, Geyer MA (1990): Apomorphine disrupts the inhibition of acoustic startle induced by weak prepulses in rats. Psychopharmacology 102:1-4

Einon D, Morgan MJ, Sahakian BJ (1975): The development of intersession habituation and emergence in socially reared and isolated rats. Dev Psychobiol 8:553-559

Gentsch C, Lichtsteiner M, Feer H (1981): Individual housing of rats causes divergent changes in spontaneous and reactive activity. Experientia 37:61-62

Gentsch C, Lichtsteiner M, Feer H (1982b): Behavioural comparisons between individually and group-housed male rats: Effects of novel environments and diurnal rhythm. Behav Brain Res 6:93-100

Gentsch C, Lichtsteiner M, Feer H (1983): Individually housed rats exceed group-housed animals in rotational movements when exposed to a novel environment. Experientia 39:1412-1414

Gentsch C, Lichtsteiner M, Kraeuchi K, Feer H (1982a): Different reaction patterns in individually and socially reared rats during exposures to novel environments. Behav Brain Res 4:45-54

Geyer MA, Swerdlow NL, Mansbach RS, Braff DL (1990): Startle response models of sensorimotor gating and habituation deficits in schizophrenia. Brain Res Bull 25:485-498

Geyer MA, Wilkinson LS, Humby T, Robbins TW (1993): Isolation rearing of rats produces a deficit in prepulse inhibition of acoustic startle similar to that in schizophrenia. Biol Psychiatry 34:361-372

Groenewegen HJ, Berendse HW, Meredith GE, Haber SN, Voorn P, Wolters JG, Lohman AHM (1991): Functional anatomy of the ventral, limbic-system innervated striatum. In Wilner P, Scheel-Kruger J (eds), The Mesolimbic System: From Motivation to Action. London, John Wiley and Sons Ltd, pp 1-39

Ison JR, Hoffman HS (1983): Reflex modifications in the do- main of startle: II. The anomolous history of a robust and ubiquitous phenomenon. Psychol Bull 94:3-17

Jones GH, Hernandez TD, Kendall DA, Marsden CA, Robbins TW (1992): Dopaminergic and serotonergic function following isolation-rearing in rats: A study of behavioural responses and post-mortem and in vivo neurochemistry. Pharmacol Biochem Behav 43:17-35

Jones GH, Marsden CA, Robbins TW (1990): Increased sensitivity to amphetamine and reward-related stimuli following social isolation in rats: Possible disruption of dopamine-dependent mechanisms of the nucleus accumbens. Psychopharmacology 102:364-372

Jones GH, Marsden CA, Robbins TW (1991): Behavioural rigidity and rule-learning deficits following social isolation in rats: Neurochemical correlates. Behav Brain Res 43:35-50

Kelly PH, Seviour PW, Iversen SD (1975): Amphetamine and apomorphine responses in the rat following 6-OHDA lesions of the nucleus accumbens septi and corpus striatum. Brain Res 94:507-522

Killcross AS, Dickinson A, Robbins TW (1994): Amphetamineinduced disruptions of latent inhibition are reinforcer mediated: Implications for animal models of schizophrenia. Psychopharmacology (In Press)

Killcross AS, Robbins TW (1993): Differential effects of intraaccumbens and systemic amphetamine on latent inhibition using an on-baseline, within-subject conditioned suppression paradigm. Psychopharmacology 110:479489

LeMoal M, Simon H (1991): Mesocorticolimbic dopaminergic network - functional and regulatory roles. Physiological Rev 71:155-234.

Lewis MH, Gluck JP, Beauchamp AJ, Keresztury MF, Mailman RB (1990): Long term effects of social isolation in Macaca mulatta: Changes in dopamine receptor function following apomorphine challenge. Brain Res 513:67-73

Lubow RE (1973): Latent inhibition. Psychol Bull 79:398-407

Mansbach RS, Geyer MA, Braff DL (1988): Dopaminergic stimulation disrupts sensorimotor gating in the rat. Psychopharmacology 94:507-514

Martin LJ, Spicer DM, Lewis MH, Gluck JP, Cork LC (1991): Social deprivation of infant rhesus monkeys alters the chemoarchitecture of the brain: I. Subcortical regions. J Neurosci 11:3344-3358

Morgan MJ, Einon D, Morris RGM (1977): Inhibition and isolation rearing in the rat: Extinction and satiation. Physiol Behav 18:1-5

Sachser N (1986): The effects of long term isolation on physiology and behaviour in male guinea pigs. Physiol Behav 38:31-39

Sahakian BJ, Burdess C, Luckhurst H, Trayhurn P (1982): Hyperactivity and obesity: The interaction of social isolation and cafeteria feeding. Physiol Behav 28:117-124.

Sahakian BJ, Robbins TW, Iversen SD (1977): The effects of isolation rearing on exploration in the rat. Animal Learn Behav 5:193-198

Sahakian BJ, Robbins TW, Morgan MJ, Iversen SD (1975): The effects of psychomotor stimulants on stereotypy and locomotor activity in socially deprived and control rats. Brain Res 84:195-205. 
Swerdlow NR, Geyer MA, Braff DL (1990b): GABAergic projection from nucleus accumbens to ventral pallidum mediates dopamine-induced sensorimotorgating deficits of acoustic startle in rats. Brain Res 532:146-150

Swerdlow NR, Keith VA, Braff DL, Geyer MA (1991): Effects of spiperone, raclopride, SCH 23390 and clozapine on apomorphine inhibition of sensorimotor gating of the startle response in the rat. J Pharmacol Exp Ther 256: $530-536$

Swerdlow NR, Masten VL, Braff DL, Geyer MA (1990a): Schizophrenic-like sensorimotor gating abnormalities in rats following dopamine infusion into the nucleus accumbens. Psychopharmacology 101:414-420

Ungerstedt U (1984): Measurement of neurotransmitter release by intracranial dialysis. In Marsden CA (ed), Mea- surement of Neurotransmitter Release In Vivo. Chichester, Wiley, pp 81-105

Weiner I, Feldon J (1987): Facilitation of latent inhibition by haloperidol. Psychopharmacology 91:248-253

Weiner I, Lubow RE, Feldon J (1988): Disruption of latent inhibition by acute administration of low doses of amphetamine. Pharmacol Biochem Behav 30:871-878

Weiner I (1990): Neural substrates of latent inhibition: The switching model. Psychol Bull 108:442-461

Winer BJ (1971): Statistical Principles In Experimental Design, 2nd ed. New York, McGraw-Hill

Zetterstrom T, Sharp T, Marsden CA, Ungerstedt U (1983): In vivo measurement of dopamine and its metabolites by intracerebral dialysis: Changes after $d$-amphetamine. J Neurochem 41:1769-1773 